\title{
¿Qué tipo de información codifica realmente un evidencial? Propuesta de una distinción conceptual entre fuente, base y modo de acceso para el reconocimiento de unidades evidenciales
}

\section{What type of information does an evidential actually encode? Proposal for a conceptual distinction between source, basis and mode of access as a new framework for the identification of evidentials ${ }^{1}$}

\author{
DÁMASO IZQUIERDO ALEGRÍA \\ GRADUN-ICS, Universidad de Navarra. Pamplona, España. \\ dizquierdo@alumni.unav.es
}

Los estudios evidencialistas más influyentes tienden a emplear los sustantivos source y evidence como palabras clave para describir el contenido transmitido por los evidenciales. Aunque estos nombres pueden designar entidades epistemológicas muy diferentes, en la bibliografía especializada no suelen explicitarse sus límites referenciales. Así, diferentes unidades y construcciones lingüísticas muy heterogéneas de diversas lenguas germánicas y romances han terminado etiquetándose como evidenciales porque los valores que transmiten pueden parafrasearse con las palabras source o evidence, aunque el semantismo de muchas de ellas en nada se parece al de unidades evidenciales inequívocas como las del tuyuca o el quechua. En este artículo introducimos una distinción conceptual (fuente, base, modo de acceso) para evitar los problemas terminológico-nocionales derivados de un uso intuitivo de los nombres sourcelfuente y evidencelevidencia en la bibliografía evidencialista centrada en idiomas europeos. Para mostrar su productividad, se ha aplicado esta distinción a los sintagmas nominales encabezados por según.

Palabras clave: evidencialidad, fuente, evidencia, modo de acceso, según.

\footnotetext{
${ }^{1}$ Este artículo se inscribe en el marco de los proyectos de investigación "Metadiscurso y lenguaje evaluativo: perspectivas teóricas y de análisis en el discurso periodístico", financiado por el Ministerio de Economía y Competitividad (FFI2012-36309), y "El discurso público: estrategias persuasivas y de interpretación", desarrollado por GRADUN (Grupo Análisis del Discurso. Universidad de Navarra) en el seno del ICS (Instituto Cultura y Sociedad) de la Universidad de Navarra. Asimismo, este trabajo ha recibido el soporte económico de una Beca del Programa de Formación del Profesorado Universitario (FPU) del Ministerio de Educación. Agradezco a Ramón González Ruiz y a Patrick Dendale su lectura del artículo y sus comentarios.
} 
In the most influential studies on evidentiality the nouns source and evidence are frequently used to refer to the values conveyed by evidentials. Although these words can designate very different epistemological notions, they are rarely defined as terms in specialized studies. Consequently, very heterogeneous linguistic items and constructions in different Germanic and Romance languages are considered evidentials because the values they convey can be paraphrased with the words source or evidence, even though many of them barely share any semantic property with true evidentials like those found in Tuyuca or Quechua. We propose a conceptual distinction (source, basis, mode of access) in order to avoid the terminological and notional problems arisen from an intuitive use of the words source and evidence in the literature on evidentiality applied to European languages. The productivity of this distinction is illustrated by a case study: the noun phrases initiated by the Spanish preposition segun.

Key words: Evidentiality, source, evidence, mode of access, según.

\section{INTRODUCCIÓN}

Como es bien sabido, la delimitación de las fronteras del concepto de evidencialidad ha sido un problema teórico que ha ocupado mucho espacio en la bibliografía especializada y ha generado posiciones claramente diferenciadas. El establecimiento de esos límites nocionales se ha concentrado en, al menos, dos focos principales:

1.1. El aspecto teórico que probablemente más influencia haya tenido en los estudios evidencialistas es la relación existente entre la evidencialidad y la modalidad epistémica. Si bien los primeros lingüistas que emplean el adjetivo evidential lo hacen para etiquetar unidades que marcan "la fuente de información en la que el hablante está basando su afirmación” (Kaye 1971: 40)², la progresiva aplicación del concepto de evidencialidad a la descripción de unidades lingüísticas de un creciente número de lenguas pronto empezó a mostrar que, en ocasiones, la referencia a un determinado tipo de fuente parece implicar la indicación de un determinado grado de compromiso epistémico por parte del locutor, en función de la fiabilidad asignada a cada fuente. Así pues, terminaron estableciéndose dos principales posturas, muy extendidas en la bibliografía (cf. Willett 1988; Dendale 1991: 47-50; Dendale y Tasmowski 2001): la evidencialidad en sentido "ancho" (in a broad sense), entendida como hiperónimo que abarca la marcación lingüística no solo de las fuentes de la información, sino también del grado de compromiso epistémico del hablante o, de forma más general, su actitud hacia el conocimiento (cf., entre otros, Chafe 1986; Biber y Finegan 1989); y la evidencialidad en sentido "estrecho" (in a narrow [er] sense), restringida a la mera indicación de las fuentes en que el hablante se basa y conceptualmente diferenciada de la modalidad epistémica. Dado que, como se ha demostrado (cf., entre otros, De Haan 1999; Aikhenvald 2004; Squartini 2004; Cornillie 2009), no existe una correlación constante y universal entre cada tipo de fuente y un determinado grado de compromiso epistémico, en

${ }^{2}$ Traducimos al español todas las citas incluidas en este artículo cuya lengua original es el inglés o el francés. 
este artículo se adopta la noción de evidencialidad en sentido "estrecho", como categoría diferenciada de la modalidad epistémica.

1.2. Otra cuestión relacionada con los límites de la evidencialidad que ha gozado de cierta difusión es la conexión que mantiene con el concepto de miratividad. Esta categoría comprende aquellas piezas que indican que la información es inesperada, nueva o sorprendente. Dado que en algunas lenguas ciertos evidenciales suelen adquirir un efecto de sentido mirativo, se ha discutido cuál es el vínculo que existe entre la evidencialidad y la miratividad (cf., entre otros, DeLancey 2001; De Haan 2012; Aikhenvald 2012). De acuerdo con la mayor parte de la bibliografía, en este artículo no se incluirá la miratividad bajo el paraguas de la evidencialidad porque, si bien ciertos evidenciales de algunas lenguas pueden adquirir contextualmente un efecto de sentido mirativo, no existe una relación biunívoca entre la evidencialidad y la miratividad desde una perspectiva interlingüística.

1.3. Como muestra este breve panorama bibliográfico, gran parte de las reflexiones acerca de la delimitación de las fronteras de la evidencialidad se han llevado a cabo por oposición a otra categoría contigua a ella con la que parece establecerse algún vínculo, como ocurre con la modalidad epistémica o la miratividad. Este mayor interés por los límites externos de la evidencialidad en comparación con otras categorías concretas parece haber dejado en un segundo plano una delineación más nítida de las fronteras del dominio conceptual que cubre la evidencialidad más allá de dicotomías específicas. Como se intenta mostrar en el presente trabajo $(\$ 2)$, parece existir cierta vaguedad terminológica en buena parte de las definiciones y descripciones de la evidencialidad, lo cual contribuye, involuntariamente, a una mayor confusión conceptual y ha abierto la puerta a la descripción de ciertas unidades del espańol y de otras lenguas como evidenciales, pese a que su semantismo se sitúa en un ámbito que tan solo puede considerarse adyacente a la evidencialidad. Teniendo en cuenta este problema, introducimos una distinción conceptual (\$3) con el fin de mejorar las deficiencias expuestas en $\$ 2$. Finalmente, aplicamos esta propuesta teórica a un grupo de unidades que ilustran con claridad la vaguedad terminológica que parece residir en muchas influyentes caracterizaciones de la evidencialidad y que, por ello, son muy representativas para la introducción de la distinción conceptual planteada en $\$ 3$ : los sintagmas iniciados por la preposición según $(\$ 4)$.

\section{Definiciones DE EVIDENCIALIDAD Y PROBLEMAS CON LOS SUSTANTIVOS SOURCE/FUENTE Y EVIDENCE/EVIDENCIA}

2.1. Más allá de la diferenciación de la evidencialidad frente a otras categorías, la mayor parte de las definiciones generales de la evidencialidad comúnmente aceptadas suelen recurrir al sustantivo source -o a sus equivalentes en otras lenguas- para especificar qué tipo de información transmite un evidencial, como se desprende de la siguiente muestra: 
(1a) Evidentials may be generally defined as markers that indicate something about the source of information in the proposition. (Bybee 1985: 184)

(1b) I here choose to consider evidentiality in the narrower sense of information source. (Willett 1988: 56)

(1c) Source of information is the semantic core of any evidential. (Aikhenvald 2004: 153$)^{3}$

(2a) La evidencialidad es el dominio semántico relacionado con la fuente de la información expresada en un enunciado. (Bermúdez 2005: 5)

(2b) La evidencialidad se define como la categoría semántica que indica las fuentes de información en las que se fundamenta el hablante, o dicho con otras palabras, especifica los distintos modos de adquisición de la información que transmitimos. (González Vázquez 2006: 15)

(3a) L'évidentialité ou le marquage des sources du savoir. (Dendale y Tasmowski 1994: título)

(3b) [...] un marquage évidentiel des énoncés, marquage par lequel nous pouvons indiquer la source ou la nature de la source d'où provient l'information transmise par les énoncés. (Kronning 2003: 131) ${ }^{4}$

Ahora bien, tal y como comenta Guentchéva (2014a: 37), "la noción de fuente de la información sigue siendo confusa”; tras la palabra source (y sus equivalentes en otras lenguas) parece ocultarse una noción que, epistemológicamente, puede resultar confusa. Si, de entre las múltiples acepciones de los nombres fuente y source en las principales obras lexicográficas del español $(4 a-c)$ y del inglés $(5 a-b)^{5}$, se seleccionan aquellas que están directamente relacionadas con la adquisición de conocimiento, se advierte que estos sustantivos tienen un significado que los faculta para designar diferentes nociones epistemológicas:

(4a) DEA: 4 Lugar o pers. de donde se obtiene información.

(4b) DLE: 8. Persona o cosa que proporciona información.

(4c) DUE: 6 Sitio de donde se obtiene una * noticia, conocimiento o información.

(5a) OED: 4.e. Un trabajo, etc., que proporciona información o "evidencia" ${ }^{6}$ (en

\footnotetext{
${ }^{3}$ Traducción: (1a) "Los evidenciales pueden definirse generalmente como marcadores que indican algo acerca de la fuente de información en la proposición". (1b) "Aquí decido considerar la evidencialidad en el sentido estrecho de fuente de información". (1c) "La fuente de información es el núcleo semántico de todo evidencial".

${ }^{4}$ Traducción: (3a) "La evidencialidad o la marcación de las fuentes del saber". (3b) "una marcación evidencial de los enunciados, marcación por la cual podemos indicar la fuente o la naturaleza de la fuente de la que procede la información trasmitida por los enunciados".

${ }^{5}$ En adelante, para una mayor claridad expositiva, se recogerán únicamente definiciones y testimonios de los sustantivos esp. fuente e ingl. source.

${ }^{6}$ Marcamos entre comillas el sustantivo evidencia en nuestras traducciones de las citas que contienen el nombre inglés evidence porque, como se expondrá (\$2.2), esta palabra genera muchas dificultades de traducción y ha
} 
especial de carácter original o primario) en relación con un determinado hecho, evento, o una serie de ellos. También, una persona que proporciona información, un informante, un portavoz.

(5b) Webster's Third New Dictionary: 2b (3): alguien que proporciona información.

Se pueden distinguir al menos cuatro tipos de referentes que resultan relevantes desde una perspectiva epistemológica:

(I) Un enunciador que suministra información verbalmente, como muestran las definiciones (4a-b) y (5a) mediante el nombre persona. Véanse, a continuación, los ejemplos (6a) y (7a), donde se señala que el referente de los sustantivos fuente y source es un ser humano cuya identidad se especifica. En (6b) y (7b) estos nombres también designan a personas que han aportado información verbalmente, pues constituyen el núcleo de sendos sintagmas nominales que desempeñan la función de sujeto de un verbo dicendi (decir, say). A juzgar por su destacada presencia en las definiciones consultadas y su alta frecuencia en las búsquedas de corpus realizadas, todo apunta a que este es el tipo de referente que con mayor asiduidad tienen fuente y source $e^{7}$ :

(6a) El hecho de que el tono sea elogioso, que no se tengan suficientemente en consideración los posibles riesgos y que la única fuente sea el médico que la realiza en la sanidad privada son factores que contribuyen a una sospecha que podría haberse evitado. (El Pais, 02/10/2011)

(6b) Fuentes de la oposición dijeron que al menos 10 personas murieron por disparos de las fuerzas de seguridad [...]. (El País, 31/12/2011)

(7a) In July, the army intelligence analyst Private Bradley Manning, the suspected source of the WikiLeaks war documents, was charged with unauthorised disclosure of US classified information. (The Times, 27/11/2010)

(7b) "There is an element of reflection going on," the source said. (The Guardian, $05 / 03 / 2011)^{8}$

\footnotetext{
dado lugar a importantes malentendidos en la bibliografía.

${ }^{7}$ En cualquier caso, no se trata de una conclusión hallada tras un estudio de corpus pormenorizado con datos cuantitativos, sino de una mera apreciación a partir de la consulta impresionista de varios corpus (no solo del corpus escogido para ilustrar las explicaciones aportadas, compuesto por los textos publicados entre 2008 y 2012 en la versión impresa de los diarios generalistas espańoles $A B C$ y El País y británicos The Guardian y The Times). Un análisis semántico detallado del sustantivo fuente y de sus equivalentes en otras lenguas fundamentado en corpus sería muy interesante, pero quedaría fuera de los límites de este trabajo.

${ }^{8}$ Traducción: (7a) "En julio, el analista de inteligencia del ejército Bradley Manning, de quien se sospecha que es la fuente de los documentos de guerra de WikiLeaks, fue acusado de divulgación no autorizada de información clasificada de EE.UU.". (7b) "«Hay un elemento de reflexión en marcha», dijo una fuente".
} 
(II) El lugar donde el locutor halla la información, referente al que se apunta en las definiciones a través de nombres como "lugar" (4a), "sitio" (4c) o incluso "cosa" (4b). La primera parte de la acepción de (5a) también se enmarca en esta dirección. Frecuentemente, ese lugar constituye una entidad de carácter discursivo (textos concretos, géneros textuales, etc.), como queda patente en los ejemplos (8a-b) y (9a). Sin embargo, este tipo de referente no siempre se corresponde con un texto o grupo de textos. En (9b), por ejemplo, se explica que a los periodistas de la $\mathrm{BBC}$ se les ha solicitado que manejen como fuente de información las redes sociales, las cuales, en sí mismas, no son entidades de naturaleza discursiva, sino aplicaciones electrónicas que contienen información tanto en forma de textos (los mensajes publicados por los usuarios, por ejemplo), como en otros soportes (imágenes, vídeos, etc.). Aun más claro es el caso de (8c), donde se informa de la prohibición de utilizar los pasillos de la Audiencia como fuente de información: las dependencias de la Audiencia son lugares de los que un periodista puede extraer abundante información no solo a partir de los textos orales y escritos que allí puede captar (diálogos, carteles, documentos oficiales, etc.), sino también a partir de un amplio abanico de estímulos sensoriales en cuya obtención no media el lenguaje (la percepción a través de la vista de las personas que se encuentran en ese lugar y de sus semblantes, gestos y movimientos, la captación de ruidos y demás sonidos de naturaleza no lingüística, etc.). Con cierta frecuencia, en este tipo de ocurrencias, fuentel source es el núcleo de un sintagma nominal con un complemento de nombre que contiene la palabra información/information, como en (8b-c) o (9b), si bien ello no es en absoluto imprescindible para que tenga esta designación, como evidencia el resto de testimonios:

(8a) Entonces emprendía una lectura extenuante de autos y sentencias, recursos, anexos, votos discrepantes, jurisprudencia. Nunca recurría a fuentes secundarias ni utilizaba resúmenes [...]. (El País, 17/12/2011)

(8b) Tal como señala acertadamente el señor Ballesteros, el artículo no indica las fuentes de donde procede la información. Por eso, propongo que se publique que las fuentes son, además del documental de Annie Leonard y el libro de la misma autora, el documental Surplus, de Erik Gandini; el documental La hora 11, de Leila y Nadia Conners; [...]. (El País, 27/02/2011)

(8c) En éste se especifica que los pasillos u otras dependencias de esos edificios «no son fuentes de información de acceso general, pues el derecho de acceso tiene un carácter instrumental, es decir, paso para llegar a aquellos locales». $(A B C$, $04 / 02 / 2010)$

(9a) Sadly for the Çelebi brothers, hardly anyone outside Turkey regards the sole account of their flights as a credible source. (The Times, 28/11/2009)

(9b) BBC news journalists have been told to use social media as a primary source of information by Peter Horrocks, the director of the BBC World Service. (The Guardian, 10/02/2010) ${ }^{9}$

${ }_{9}^{9}$ Traducción: (9a) "Lamentablemente para los hermanos Çelebi, casi nadie fuera de Turquía considera el mero 
(III) Quizá con menor asiduidad, los indicios concretos en los que una persona se basa para obtener nuevo conocimiento. Aunque este tipo de referente no está claramente representado en las definiciones de (4-5), se pueden hallar testimonios ocasionales que se ajustan a esta descripción: en (10a), de manera humorística, el autor del texto afirma que la política española Ana Mato es naturista apoyándose en el aspecto de su melena, el cual categoriza como su fuente. La apariencia de su cabello es el indicio sobre el que el emisor se basa para desencadenar un proceso inferencial, deliberadamente extravagante, cuyo resultado es la adscripción sarcástica de esta política al naturismo: si su cabello no presenta el aspecto de aquel que ha sido tratado en una peluquería, es posible que sea ella misma quien se lo haya teńido y que exista una razón para ello (el rechazo de productos químicos por seguir los principios del naturismo).

(10a) Liviana, angelical: Ana Mato es, sencillamente, una naturista, una seguidora del eminente especialista argentino doctor Scolnik. ¿Que cuáles son mis fuentes? ¡Su melena! ¿Es que no ven que se la tiñe con camomila casera? Esa mujer jamás frecuentó una peluquería ni acudió a añagazas químicas. (El País, 28/06/2012)

En (10b), el sustantivo fuente, que está coordinado con el nombre marcador, parece designar el indicio o elemento en que se basa el locutor del enunciado reproducido, el expresidente español José Luis Rodríguez Zapatero, para juzgar que su partido político es merecedor de una victoria electoral. Señalar que alguien merece algo requiere, epistemológicamente, disponer de unos conocimientos previos sobre sus cualidades y acciones, que, contrastados con ciertos criterios y valores, conducen a una evaluación cuyo resultado es la conclusión de que esa persona es o no merecedora de algo.

(10b) “ $\mathrm{i} M e r e c e m o s$ ganar y ganaremos!", ha dicho este pasado fin de semana, en Sevilla, José Luis Rodríguez Zapatero. No constan la fuente o el marcador que le atribuye el mérito a un socialismo que, precisamente en Sevilla, debiera tener la prudencia del silencio después de que más de 600 millones de euros estén en el alero del "caso Mercasevilla", una chorizada de tomo y lomo. ( $A B C$, $15 / 02 / 2011)$

Aunque el tipo de referente (III) parece próximo al de (II), estos no son coincidentes: el tipo de referente (II) se identifica con el lugar en que se ubican los indicios específicos que posibilitan al hablante obtener una información, mientras que el tipo de referente (III) designa directamente esos indicios concretos que permiten el acceso a nuevo conocimiento.

informe de sus vuelos como una fuente creíble". (9b) "Peter Horrocks, director del Servicio Mundial de la BBC, pidió a los nuevos periodistas de la $\mathrm{BBC}$ que utilizaran los medios de comunicación sociales como la fuente primaria de información". 
(IV) Por último, también con poca frecuencia, un proceso de obtención y difusión de información. En (11), por ejemplo, el emisor caracteriza como una fuente de información la transmisión oral de rumores ("el boca a boca"). En (12), junto a este canal epistemológico ("word of mouth"), también se categoriza como una fuente "la investigación en Internet" ("online research"). Claramente, en ambos casos este sintagma designa un proceso epistemológico (referente IV) y no un enunciador (referente I), un lugar (referente II, ya que no es Internet lo que se considera una fuente, sino las pesquisas llevadas a cabo a partir de la información publicada en línea), ni, por fin, un indicio (referente III, pues no se dice que la fuente sea la información hallada en Internet, sino el proceso de investigación que con ella se efectúa) ${ }^{10}$.

(11) El boca a boca entre los vecinos era a esas horas la mejor fuente de información, y la verdad es que las noticias que se trasladaban eran cada minuto peores. (ABC, 12/05/2011)

(12) Word of mouth and online research are two of the most important sources of information for prospective postgraduates. (The Guardian, 16/03/2010) ${ }^{11}$

Pese a esta diversidad de referentes de naturaleza epistemológica, en la bibliografía evidencialista suelen darse por presupuestos los límites designativos de las palabras source y fuente, dado que, en la gran mayoría de los casos, estas son constantemente empleadas sin que previamente hayan sido objeto de una definición. Por ello, no es de extrañar que también en los estudios evidencialistas estos nombres se utilicen para hacer referencia a entidades epistemológicamente muy heterogéneas. Véanse las citas de (13), (14), (15) y (16), donde designan, respectivamente, un tipo de referente (I), (II), (III) y (IV).

(13a) John indicates that his information source is Bill Fraenkel. (Whitt 2010: 153) ${ }^{12}$

(13b) [...] la fuente o persona con autoridad y conocimiento suficiente para utilizarse como fuente de la información, es, en este caso, el alcalde de Calvià en 1990. (Rodríguez Ramalle 2008: 813)

(14a) Although in (4) the access to the source of information, i.e. newspaper article(s), can hardly be considered restricted to the speaker, the speaker's conclusion is

\footnotetext{
${ }^{10}$ Este sucinto análisis de los sustantivos fuente y source no pretende ser un examen detallado de su semántica, sino solamente una recopilación de sus acepciones relacionadas con el acceso a nuevo conocimiento y de sus diferentes tipos de referentes epistemológicos. En cualquier caso, en los cuatro tipos de referentes descritos, es indudable que, semánticamente, existe un nexo de unión, que se corresponde con la idea general de 'origen' de la información, pues ese origen puede hallarse en un ser animado (I), un lugar (II), un indicio concreto (III) o bien un proceso epistemológico (IV).

${ }^{11}$ Traducción: (12) "El boca a boca y la investigación en Internet son dos de las fuentes de información más importantes para los futuros alumnos de posgrado".

${ }^{12}$ Traducción: (13a) "John indica que su fuente de información es Bill Fraenkel”.
} 
not necessarily shared with other people. (Cornillie 2007: 25) ) $^{13}$

(14b) [...] por su parte, la información que proviene de una fuente indirecta -del discurso de otra persona- al ser menos verificable, se sitúa en un rango inferior dentro de la escala de la confiabilidad. (Estrada 2008: 9)

(15a) These evidentials are common in first person contexts as well, normally indicating an internal or external feeling as the source of knowledge. (Curnow 2002: $\$ 4.4)^{14}$

(15b) [...] como se ve en el comentario que el periodista introduce acto seguido, aclara que su fuente de información no es lo que él mismo ha presenciado sino lo que ha leído en la prensa. (Marcos Sánchez 2016: 169)

(16a) Nevertheless, such elements can still be classed as indirect evidentials as they are infelicitous when the speaker has direct evidence for $p$ and because they point to a mental process as the source of information for $p$. (Faller 2011: 672) ${ }^{15}$

(16b) El futuro indica, por tanto, que en el momento del habla, el hablante no tiene otra fuente mejor que sus propios procesos internos para presentar un evento. (Escandell 2010: 23)

2.2. Otro nombre muy empleado en la bibliografía anglófona para definir la evidencialidad y que podría considerarse la alternativa a los problemas de fuente y source es la palabra de la que proceden los propios términos evidentiality y evidential: el sustantivo evidence:

(17a) Evidentials express the kinds of evidence a person has for making factual claims. (Anderson 1986: 273)

(17b) [E]videntiality [...] deals with the evidence the speaker has for his or her statement. (De Haan 1999: \$6) ${ }^{16}$

No obstante, el uso de la palabra evidence también plantea dificultades. En los estudios evidencialistas, este nombre suele emplearse como sinónimo de indicio, esto es, para designar aquellos datos en los que el hablante se apoya para acceder a nueva información, de

\footnotetext{
${ }^{13}$ Traducción: (14a) "Aunque en (4) difícilmente puede considerarse que el acceso a la fuente de información, i.e. uno o varios artículos periodísticos, esté restringido al hablante, la conclusión del hablante no necesariamente es compartida con otras personas".

${ }^{14}$ Traducción: (15a) "Estos evidenciales también son habituales en contextos de primera persona, normalmente indicando una sensación interna o externa como la fuente de información".

${ }^{15}$ Traducción: (16a) "No obstante, estos elementos pueden seguir clasificándose como evidenciales indirectos, dado que su uso no es satisfactorio cuando el hablante tiene "evidencia" directa de $p$ y porque apuntan a un proceso mental como fuente de información de $p$ ".

${ }^{16}$ Traducción: (17a) "Los evidenciales expresan los tipos de "evidencia" que una persona tiene para hacer afirmaciones factuales". (17b) "La evidencialidad se ocupa de la "evidencia" que tiene el hablante de su afirmación".
} 
manera similar al tipo de referente (III) de fuente (cf. supra \$2.1). Como señala Aikhenvald (2004: 4-5), este uso difiere del significado que evidence tiene comúnmente fuera de cualquier terminología, el cual podría describirse con estas palabras: 'aquello que manifiesta o hace evidente' (cf. OED: s.v. II), a menudo con un valor probatorio o demostrativo.

Esta diferencia de significado se produce de forma aún más acusada en las lenguas romances, donde el uso del sustantivo evidencia -o de sus cognados- como equivalente de indicio es muy marginal fuera de los estudios evidencialistas y parece constituir un préstamo semántico, ya que el nombre inglés evidence y sus cognados en las lenguas romances pueden considerarse, en realidad, falsos amigos. Esta reflexión es uno de los motivos por los que varios autores del ámbito francófono rechazan los términos évidentialité y évidentiel en francés, pues, en su opinión, sugieren que un evidencial marca que la información transmitida es evidente o que ha sido probada, cuando ciertamente no es este el ámbito nocional que la evidencialidad cubre. Véanse, a este respecto, las justificaciones de, entre otros, Guentchéva (1996, 2014a, 2014b), Lazard (1999) o Tournadre (2004: 52), quienes, en su lugar, prefieren el adjetivo médiatif ${ }^{7}$. Este riesgo, de hecho, se ha materializado en la bibliografía en español, donde se pueden localizar numerosos estudios representativos que incluyen los términos evidencialidad y evidencial para hacer referencia, exclusiva o fundamentalmente, a unidades lingüísticas que califican la información transmitida como evidente:

(18a) El resto de las partículas epistémicas "evidenciales" admiten la combinación con que (claro que lo sabe; evidentemente que sí; ciertamente que lo ha dicho, etc.). Se distinguen entre sí esencialmente en virtud del tipo de "evaluación" de la "evidencia" que reflejan. (Martín Zorraquino 1999: 39)

(18b) [...] Distinguiremos entre los que se indican:

-evidencialidad o reafirmación: evidentemente, ciertamente...

-duda, posibilidad, probabilidad: probablemente, posiblemente, quizás, tal vez -origen de las informaciones, o marcas de enunciador: al parecer, por lo visto, personalmente [...] (Fuentes Rodríguez 2007: 35)

(18c) [...] y los adverbios evidenciales, por ejemplo, naturalmente, evidentemente, lógicamente, obviamente, caracterizados por denotar un grado de compromiso con la verdad de la proposición mucho mayor que los epistémicos de duda y posibilidad, pues con ellos el hablante otorga credibilidad a lo que dice [...]. (Rodríguez Ramalle 2008: 812)

(18d) En sus apariciones como adverbio oracional es un adverbio modal, epistémico evidencial. Estos adverbios se emplean para expresar que el hablante considera evidente su enunciado. (Acín 2014: 188)

\footnotetext{
${ }^{17}$ Aunque este no es el único motivo por el que algunos autores adoptan esta etiqueta. Un mediativo marca que la información que el locutor transmite "no se ha obtenido a partir de una experiencia personal" (Guentchéva 1996: 11), es decir, la mediatividad excluye la evidencialidad directa.
} 
Para esquivar este problema, conviene evitar la palabra evidencia en las definiciones de evidencialidad en español ${ }^{18}$. No en vano, en el estudio de los adverbios oracionales y, en general, de los operadores modales del español, desde mucho antes de la popularización de la etiqueta evidencial, era frecuente el uso del nombre evidencia para glosar el significado de unidades como claro o naturalmente, en la medida en que califican como evidente el contenido de la proposición (cf., entre otros, Barrenechea 1979: \$2.2.1; Fuentes Rodríguez 1995; Martín Zorraquino y Portolés 1999: \$\$63.6.2.3-4). Cuando el término evidencial empieza a permear la bibliografía en español y sus límites todavía resultan muy difusos, el paso de la denominación "marcadores de evidencia" a "marcadores evidenciales" se produce de forma más o menos rápida e inconsciente, como ilustran las citas de (18).

Además de los riesgos de la vinculación del sustantivo esp. evidencia al adjetivo evidente, la manera en que su cognado inglés evidence es empleado en la bibliografía evidencialista también revela una designación epistemológicamente difusa. Muestra de ello es la frecuente coaparición de evidence y source como equivalentes funcionales (19) o el recurrente empleo del sintagma source of evidence, cuya extensión parece vaga (20):

(19a) Evidentiality concerns the indication of the source or kind of evidence speakers have for their statements. (Van der Auwera y Plungian 1998: 85)

(19b) [...] there seems to be consensus that evidential meaning is definable with reference to this notion or to the notion of information source or evidence. (Boye y Harder 2009: 14)

(20a) Evidentiality refers to the grammatical marking of the source of evidence for a proposition. (DeLancey 2001: 369)

(20b) Evidentiality proper is understood as stating the existence of a source of evidence for some information; [...]. (Aikhenvald 2003: 1) ${ }^{19}$

2.3. Si partimos de una concepción de la evidencialidad como marcación lingüística de la fuentelsource o evidence de la información, similar a la que se desprende de buena parte de las caracterizaciones más difundidas, en las que estos sustantivos parecen emplearse de forma imprecisa desde un punto de vista epistemológico, algunas unidades lingüísticas del

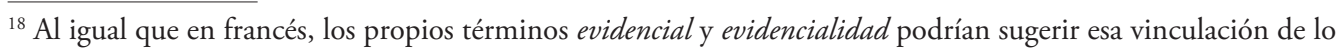
evidencial con la expresión de lo evidente, pero, a diferencia del sustantivo evidencia, presentan la ventaja de que no se emplean en castellano fuera de la terminología y su uso técnico es ya es ampliamente conocido entre los especialistas, por lo que resultaría muy difícil implantar otros términos que lograran reemplazarlos.

19 Traducción: (19a) "La evidencialidad tiene que ver con la indicación de la fuente o del tipo de "evidencia" que tienen los hablantes de sus afirmaciones". (19b) "[...] parece haber consenso en cuanto a que el significado evidencial puede definirse haciendo referencia a esta noción o a la noción de fuente de información o evidencia". (20a) "La evidencialidad se refiere a la marcación gramatical de la fuente de "evidencia" de una proposición". (20b) "Se entiende que la evidencialidad propiamente dicha expresa la existencia de una fuente de "evidencia" de cierta información".
} 
español pueden terminar considerándose evidenciales, pese a que el tipo de información que codifican diste mucho de la detectada en lenguas con evidencialidad gramaticalizada. Una unidad muy representativa para ilustrar este riesgo es la preposición tradicionalmente considerada "imperfecta" o "dudosa" según ${ }^{20}$. Entre los diferentes valores que esta pieza (y sus equivalentes en otras lenguas, como fr. selon y d'après ${ }^{21}$ ) puede transmitir cuando introduce un sintagma nominal, suele mencionarse un valor de "origen" (Coltier 2003; Féron y Coltier 2008; Thuillier 2012: 21-24) o de "fuente" de la información (Charolles 1987; Pavón Lucero 1999: \$9.2.5.1; Marque-Pucheu 1999; Schrepfer-André 2005: 16-17; Borillo 2005: 40; Maldonado y De la Mora 2015; López Martín 2017; Llopis 2018), que es el que parece primar en ocurrencias como estas ${ }^{22}$ :

(21) Según Montoro, el Gobierno "ha reconocido la gravedad de la situación económica" generada por la "inactividad" del presidente del Gobierno y del vicepresidente Solbes en los últimos cuatro años. (El País, 19/04/2008)

(22) Según la Constitución, son las propias Cámaras las que fijan la asignación económica de diputados y senadores. (ABC, 19/09/2012)

(23) [...] Serrán no puede jugar según el reglamento: un canterano no puede hacerlo en las últimas cuatro jornadas si no suma diez partidos oficiales durante el curso, o los cinco anteriores de forma consecutiva, o son menores de 23 años. (El Pais, 07/05/2008)

De hecho, con base en ejemplos similares a estos, algunos autores emplean las etiquetas evidencial y mediativo para describir el valor de según (Ferrari y Gallardo 1999: 73; González Vergara 2011: 149-150; López Izquierdo 2013; Maldonado y De la Mora 2015; López Martín 2017; Llopis 2018) o de sus equivalentes en otras lenguas (Fox 2001: 171172; Wiemer 2010: 107-109; Pietrandrea 2007: 52), en especial en francés (cf. Dendale y

\footnotetext{
${ }^{20}$ Como es sabido, según se interpreta como un miembro dudoso o imperfecto dentro del listado tradicional de preposiciones del español porque presenta varias peculiaridades. Tal y como resume Márquez Guerrero (2009: 440), "tiene carácter tónico y no impone el caso oblicuo a los pronombres que toma como término (vid.: según tú). Además, puede introducir oraciones (como las conjunciones) sin necesidad de ir acompañada de la conjunción que («Según lo vayamos viendo, iremos actuando»); por otra parte, aparece aislada en ciertos contextos, como los adverbios: «Todavía no sé si iré a la playa, según»".

${ }^{21}$ En la lingüística hispánica la semántica de según ha despertado un interés menor y más tardío (cf. Morera 1988: 438-440; Pavón Lucero 1999: \$9.2.5.1; De Bruyne 1999: \$10.18.3; Márquez Guerrero 2009; NGLE: \$29.2e-g; López Izquierdo 2013; López Martín 2017; Llopis 2018) del que han gozado en el ámbito francófono las preposiciones análogas selon y d'après. Por ello, también hemos atendido a las descripciones de estas dos últimas piezas. Aunque también existen diferencias entre esp. según y fr. selon y d'après, aquí solo hemos atendido a sus similitudes, pues no adoptamos un enfoque contrastivo.

${ }^{22}$ No es este el único valor de según: también puede parafrasearse como 'dependiendo de' o 'en función de' (cf. NGLE: \$29.2e): “Consumo por necesidad y según el precio” (El Pais, 11/08/2011). No atenderemos a este valor, dado que no presenta ninguna relación con la evidencialidad.
} 
Tasmowski 1994: 5; Marque-Pucheu 1999; Coltier y Dendale 2004: 596; Schrepfer-André 2005: \$1.2; Borillo 2005; Kim 2009; Miche 2015: 234-237).

Ahora bien, aunque fácilmente pueden emplearse las palabras fuente y source para glosar el valor de los SSNN introducidos por según en (21-23) -así como evidence en el caso de (22-23)-, existen diferencias reseñables entre estos ejemplos en cuanto al proceso epistemológico efectuado por el locutor. En (21), según introduce un sintagma que designa a un enunciador que ha verbalizado la información contenida en la proposición a la que afecta. En otras palabras, si atendemos a los tipos de referentes que más arriba hemos indicado que pueden tener los sustantivos fuente y source desde una perspectiva epistemológica (\$2.1), en (21) el SN encabezado por según se corresponde con un tipo de referente (I). En (22), el locutor recurre a según para señalar cuál es el lugar en que se ubica la información comunicada (la Constitución española), lo cual concuerda con el tipo de referente (II). En (23), por fin, pese a que según, al igual que en (22), introduce un sintagma que tiene un referente de naturaleza discursiva (el reglamento), el locutor no se ha limitado a extraer de él el contenido de la proposición a la que este sintagma modifica: es evidente que en un reglamento cuyo fin es sentar las bases generales de la competición no se expone directamente si un deportista concreto tiene permitido jugar en una situación específica; por el contrario, el locutor ha debido aplicar los criterios pertinentes que se exponen de forma explícita en el reglamento a las circunstancias de un individuo concreto.

\section{Propuesta de una distinción Conceptual entre fuente, base Y modo de aCCESO}

Tal y como hemos mostrado ( $\$ \$ 2.1-2.2)$, las palabras ingl. source y evidence y esp. fuente y evidencia son capaces de designar entidades muy diversas desde un punto de vista epistemológico, lo cual también ocurre en la bibliografía evidencialista, donde rara vez se establecen sus límites referenciales como términos especializados. No es de extrańar, pues, que ciertas unidades lingüísticas con un semantismo muy diferente al de aquellos morfemas que forman parte de sistemas verdaderamente evidenciales hayan terminado analizándose como evidenciales. Un buen ejemplo de ello, como se acaba de explicar (\$2.3), es el caso de los sintagmas nominales iniciados por la preposición según, cuyo estatus aparentemente evidencial se sustenta en el hecho de que los valores que transmiten pueden glosarse con las palabras source y fuente $\mathrm{y}$, en menor medida, también evidence y evidencia, pese a que estos sintagmas designan, en realidad, referentes epistemológicamente muy heterogéneos que exceden los límites de la semántica evidencial.

Por lo general, este halo de vaguedad respecto a los límites referenciales de las palabras fuente y source ha pasado desapercibido en la bibliografía, aunque también se documentan estudios en los que se sugiere la existencia de cierta ambigüedad en el nombre source: "si la noción de evidencialidad da lugar a tantos debates [...] es porque la noción de fuente de información recibe interpretaciones divergentes" (Guentchéva 2014b: 66); de forma excepcional, algunos autores han llegado a esbozar algunas distinciones conceptuales 
dicotómicas para tratar de esclarecer de manera más precisa cuáles son las diferentes nociones que las palabras source y fuente parecen capaces de designar (cf. Botne 1997: 523-524; Squartini 2001: \$2; Bednarek 2006: 639; Tournadre y LaPolla 2014).

Inspirados en estas reflexiones, proponemos establecer una distinción más compleja entre tres nociones epistemológicas que parecen solaparse en un uso amplio de source y fuente y, en menor medida, de evidence y evidencia: fuente, base y modo de acceso a la información. A nuestro juicio, es la tercera de las tres la que atañe de manera nuclear a la evidencialidad (cf. infra $\$ 3.4$ ), mientras que las otras dos serían secundarias en la marcación evidencial.

3.1. En general, parece existir un acuerdo tácito en la bibliografía evidencialista en que, cuando un hablante utiliza un evidencial, normalmente está aportando información acerca del proceso epistemológico que ha efectuado él mismo y no otro enunciador. Ahora bien, esa información a la que ha accedido el locutor de una manera concreta ha podido ser creada por diferentes personas. Una posibilidad es que sea el propio locutor quien la haya originado, bien sea de forma directa, a través de la constatación de ese hecho por medio de los sentidos (evidencialidad directa), como ocurre en (24a), bien sea de forma indirecta, tras efectuar una inferencia basada en diferentes tipos de datos (evidencialidad inferencial), como ilustra (24b). También es posible que, como en (24c), sea otro enunciador quien haya creado esa información, y que el locutor haya accedido a ella por medio de un enunciado emitido por aquel, de cuyo contenido simplemente se hace eco (evidencialidad reportativa).

Sistema evidencial del quechua huanca (Floyd 1997: 71; Aikhenvald 2004: 43):

(24a) Chay-chruu-mi achka wamla-pis walashr-pis alma-ku-lkaa-ña

'Muchas muchachas y muchachos estaban nadando' (los vi)

(24b) Daañu pawa-shra-si ka-ya-n-chr-ari

'Debe de estar completamente destruido' (infiero)

(24c) Ancha-p-shi wa'a-chi-nki wamla-a-ta

'Haces llorar mucho a mi hija' (me han contado)

Dendale (1991: 40) distingue esta noción de "creador de la información" de forma expresa, a la que asigna el marbete agente epistémico: "El agente epistémico es aquel que crea una información o de quien se toma una información". Asimismo, una parte de la bibliografía evidencialista, cuyo origen se remonta a Frawley (1992: 412-419), parece restringir el uso del sustantivo source para designar quién ha creado la información, si bien no se suele recurrir al verbo crear para definir este término y se prefiere hacerlo equivaler al sintagma centro epistémico. Desde este enfoque, Frawley identifica dos tipos de fuentes: el locutor (SELF) u otro enunciador (OTHER) y sienta las bases para un modelo de organización interna de la evidencialidad basada en el tipo de fuente, entendida de esta manera.

A partir de estas reflexiones, proponemos restringir el uso del nombre fuente para designar a la persona que se ha encargado del proceso de creación de la información. Adviértase que, al recurrir al verbo crear, no identificamos la noción de fuente con aquella 
persona que, en la realidad, por primera vez originó una determinada información, algo que sería muy difícil de detectar. Para aclarar este punto, planteemos la siguiente situación a modo de ejemplo: Juan emite el enunciado de (25a) dirigiéndose a Pedro y este último, a su vez, basado en dicho enunciado, emite el que se aporta en (25b):

(25a) Juan le dice a Pedro: "Está nevando en Bruselas".

(25b) Pedro le dice a Ana: "Según Juan, está nevando en Bruselas".

En (25b), consideramos que Juan es la fuente de la información contenida en la proposición, tal y como se marca mediante el sintagma según Juan. Sin embargo, ello no implica que Juan sea necesariamente la persona que por primera vez haya creado esa información en una cadena epistemológica: de hecho, es posible que Juan no haya accedido a la información directamente (porque, por ejemplo, se encuentra en Bruselas y ve cómo está nevando), sino porque su amigo Andrés, con el que acaba de hablar por teléfono, se lo ha contado. En ese caso, pese a que, en realidad, Juan basa su conocimiento únicamente en el testimonio de Andrés, no juzgamos que, lingüísticamente, sea Andrés la fuente, entendida tal y como se ha descrito en estas líneas, ya que Juan asume como propio el testimonio de su amigo y, al transmitir esa información a otra persona, lo hace mediante un enunciado declarativo que carece de cualquier referencia a otro agente epistémico, asumiendo así la responsabilidad de ella (25a); si Juan no le ha dicho a Pedro en otro enunciado por qué sabe que está nevando, difícilmente Pedro podrá detectar la existencia de otra fuente anterior de la que Juan ha obtenido la información que le está transmitiendo.

Aunque, como se ha mostrado (cf. supra \$2.1), fuente es un sustantivo que se ha concebido de formas muy diversas y su adopción en esta propuesta podría conllevar ciertos riesgos, entendemos que la definición explícita de los límites que aquí asignamos a esta etiqueta neutraliza esos peligros.

3.2. Otra dimensión epistemológica relevante es lo que aquí, inspirados en Bednarek (2006: 639), etiquetamos como base de la información. Dado que es imposible obtener información ex nibilo, todo hablante que accede a nuevo conocimiento lo hace basándose en algún tipo de información. Epistemológicamente, esas bases pueden pertenecer a tres grandes grupos: bases sensoriales (estímulos captados por los sentidos), enunciativas (el contenido de enunciados emitidos por otros hablantes) o cognitivas (datos almacenados en la memoria del hablante a los que accedió en el pasado de una manera determinada; ese modo de acceso ya ha dejado de ser relevante e incluso puede haberse olvidado ${ }^{23}$.

\footnotetext{
${ }^{23}$ A estos tres tipos principales de bases cabría añadir las bases internas, sintagma que empleamos para referirnos a aquellos estados fisiológicos y psicológicos como el dolor, el cansancio o la tristeza de los que tenemos conocimiento directo cuando los estamos experimentando y en cuya detección no interviene ninguno de los cinco sentidos, motivo por el que no tienen cabida entre las bases sensoriales. Se trata, pues, de bases esencialmente privativas, ya que nunca dos personas pueden acceder a una misma base interna de manera estrictamente directa: solo yo puedo experimentar el dolor de espalda que estoy sufriendo o la sensación de cansancio que estoy sintiendo; aunque puedo imaginarme qué es lo experimentan otras personas cuando les
} 
3.3. La tercera dimensión epistemológica es aquella que describimos como propiamente evidencial: el modo de acceso a la información. Siguiendo las taxonomías clásicas de tipos de evidencialidad (Anderson 1986; Willett 1988; Aikhenvald 2004), identificamos tres modos de acceso principales: directo (constatación directa a través de los sentidos o experimentación de sensaciones internas), reportativo (acceso a la información mediante el testimonio de otro enunciador, del que el hablante tan solo se hace eco) e inferencial (creación de una nueva información como resultado de un proceso inferencial efectuado a partir de una serie de bases), con posibilidad de ulteriores distinciones dentro de cada modo de acceso.

No existe una correspondencia directa entre cada tipo de base y cada modo de acceso: una base sensorial no supone necesariamente un modo de acceso directo, ni una base enunciativa un modo de acceso reportativo, y tampoco el modo de acceso inferencial se sustenta exclusivamente en bases cognitivas. Por el contrario, una base sensorial (la constatación a través de la vista de que el asfalto está mojado) puede llevar al hablante de forma directa al hecho que está constatando (el asfalto está mojado) o bien de forma inferencial a un hecho que no ha presenciado y que infiere por sus efectos (ha llovido). Asimismo, una misma base enunciativa (Juan dice: Ana no viene a la fiesta) puede suscitar un modo de acceso reportativo (si solamente se almacena el contenido de esa base enunciativa como procedente de otra persona y así se verbaliza ulteriormente al decir, por ejemplo, Según Juan, Ana no viene a la fiesta) o un modo de acceso inferencial (si ese enunciado de Juan desencadena una inferencia sobre, por ejemplo, la actitud de Ana: Por lo visto, Ana está enfadada).

A diferencia de los modos de acceso directo y reportativo, sería importante poner de relieve que el modo de acceso inferencial siempre requiere, como mínimo, dos bases y al menos una de ellas ha de ser una base cognitiva. En efecto, sería imposible crear una nueva información a partir de una base sensorial o enunciativa a la que se está teniendo acceso en ese momento si se careciera de todo conocimiento previo con el que poner en relación esas bases sensoriales o enunciativas. En ese caso, el hablante solo podría constatar aquello que está percibiendo mediante los sentidos (evidencialidad directa), o bien hacerse eco del contenido de un enunciado (evidencialidad reportativa), pero sería incapaz de originar nueva información relacionando una base sensorial o enunciativa con unas bases cognitivas ya almacenadas (por ejemplo, cuando una persona está enfadada con otra, normalmente no desea acudir a un lugar donde sabe que va a coincidir con ella; por ello, es plausible pensar que Ana está enfadada y que esto explica su ausencia en la fiesta).

3.4. La razón por la que juzgamos que el parámetro nuclear para definir una pieza como evidencial es el modo de acceso depende de las oposiciones evidenciales existentes en las lenguas que disponen de evidencialidad gramatical. El quechua huanca, por ejemplo, distingue entre modo de acceso directo (24a), inferencial (24b) y reportativo (24c) (\$3.1):

duele la espalda o sienten fatiga, siempre será un dolor o un cansancio diferente cuya experimentación, en términos epistemológicos, me estará siempre vedada. 
(24a) Chay-chruu-mi achka wamla-pis walashr-pis alma-ku-lkaa-ña 'Muchas muchachas y muchachos estaban nadando' (los vi)

(24b) Daañu pawa-shra-si ka-ya-n-chr-ari 'Debe de estar completamente destruido' (infiero)

(24c) Ancha-p-shi wa'a-chi-nki wamla-a-ta 'Haces llorar mucho a mi hija' (me han contado)

La fuente es en (24c) otro enunciador, mientras que (24a) y (24b) tienen en común que la información ha sido creada por el locutor (es decir, este es la fuente). La base tampoco es el parámetro fundamental que opone los enunciados de (24), ya que, aunque (24a) y (24c) muestran restricciones respecto a la base (pues esta debe ser, respectivamente, sensorial y enunciativa), en (24b), en cambio, el morfema evidencial es compatible con cualquier tipo de base: la inferencia del hablante puede sustentarse en bases sensoriales, enunciativas y cognitivas. El único de los tres parámetros epistemológicos propuestos en este artículo que opone a los morfemas evidenciales del quechua huanca es el modo de acceso: el hablante accede de diferente manera a la información si ha percibido por los sentidos aquello que está comunicando, si lo ha inferido a partir de ciertas bases o si lo ha conocido por el testimonio de otra persona. Las oposiciones evidenciales del quechua huanca ilustran que el modo de acceso es el parámetro más básico que vertebra los sistemas evidenciales.

Somos conscientes de que podría parecer que otros sistemas evidenciales más complejos no se ajustan a esta afirmación. No obstante, consideramos que se trata de un desajuste únicamente aparente y que, por tanto, con base en la observación de sistemas evidenciales muy diversos, desde los más simples hasta otros con un gran número de oposiciones, sigue siendo válida la afirmación de que una unidad lingüística, para ser considerada evidencial, debe marcar ineludiblemente el modo en que se ha accedido a la información. Ello no excluye, en cualquier caso, que un evidencial pueda, además, transmitir información adicional relacionada con la fuente, la base o cualquier otro parámetro adyacente, pero esto último no es necesario para su catalogación como evidencial.

En Aikhenvald (2004: 23-66), obra que sigue siendo aquella que recoge de manera sistemática y homogénea un mayor número de sistemas evidenciales en las lenguas del mundo, no se registra ningún paradigma en el que el parámetro básico de oposición se corresponda con lo que en este trabajo hemos denominado base y fuente: en efecto, en un sistema cuyo parámetro básico fuera el tipo de base y no el modo de acceso a la información, la unidad lingüística especializada en la marcación de una base visual podría aplicarse tanto a situaciones en que el modo de acceso fuera directo como aquellas en que la información transmitida hubiera sido el producto de una inferencia, siempre y cuando la base que desencadenase este proceso epistemológico fuera visual. Del mismo modo, si el parámetro fuente fuera el que gobernara las oposiciones dentro de un sistema evidencial, este debería contar con dos unidades: una que codificara que la fuente es el propio locutor y que se emplearía tanto en aquellos casos en que la información se ha obtenido de forma directa como inferencial; y una segunda unidad que codificaría que la fuente es otro enunciador, 
restringida para estados de cosas a los que el locutor ha accedido de manera reportativa. No obstante, ninguno de los numerosos sistemas evidenciales recopilados por Aikhenvald (2004) en su detallado catálogo encaja en tales modelos. Lamentablemente, no disponemos aquí del espacio suficiente para discutir esta cuestión con el detenimiento oportuno, labor que llevamos a cabo en otro trabajo (Izquierdo Alegría 2016: 171-187). Remitimos, pues, a Aikhenvald (2004: 23-66) para más ejemplos de sistemas evidenciales muy variados de otras lenguas que, sin embargo, siguen presentando el parámetro modo de acceso como parámetro básico del paradigma.

Algunas caracterizaciones de la evidencialidad parecen poner de relieve el parámetro que aquí etiquetamos como modo de acceso, elección que, con base en las explicaciones que se han aportado, parece más acertada que el uso de sustantivos como fuente/source o evidencialevidence. He aquí algunos ejemplos de este tipo de definiciones (las traducciones son nuestras):

(26a) El denominador común de todos estos puntos de vista es que la evidencialidad es el medio lingüístico para indicar cómo ha obtenido el hablante la información en la que basa una aserción. (Willett 1988: 55)

(26b) Nos parece que no podemos sino ganar en claridad si reservamos el término modalidad para la expresión de la actitud del locutor y evidencialidad para la expresión del modo de creación y/o recogida de la información, [...]. (Dendale y Tasmowski 1994: 4)

(26c) Se podría decir que en tales casos los hablantes indican de qué manera han accedido a la información a la que se refieren en una determinada situación de habla. (Plungian 2010: 17)

\section{Acerca de la utilidad de la distinción propuesta para el reconocimiento de EVIDENCIALES: EL CASO DE SEGÚN}

La distinción entre fuente, basey modo de acceso a la información (\$3) resulta de gran utilidad para diferenciar entre aquellas unidades lingüísticas que, desde una perspectiva nocional, pueden considerarse evidenciales (en la medida en que indican nuclearmente cómo se ha obtenido la información que se está comunicando) y otras piezas únicamente para-evidenciales (que, si bien transmiten un contenido de carácter epistemológico, no señalan por sí mismas cuál es el modo en que se ha accedido a la información, sino que tan solo aportan datos sobre otros parámetros contiguos como la fuente o las bases manejadas).

Como ya se ha adelantado $(\$ 2.3)$, una unidad lingüística muy transparente para mostrar los riesgos de una definición de la evidencialidad anclada en una delimitación imprecisa del concepto de fuente/source o evidencialevidence es la preposición "dudosa" según seguida de un SN que señala el "origen" o la "fuente" de la información. Si aplicamos la 
distinción entre fuente, base y modo de acceso, ninguno de los SSNN introducidos por la preposición según podría etiquetarse como propiamente evidencial. En función del tipo de referente designado por cada $\mathrm{SN}$, cabría distinguir dos posibilidades fundamentales, consecuentes con algunas clasificaciones propuestas en la bibliografía francófona a propósito de la preposición análoga selon (cf. Coltier 2003: 125-130; Coltier y Dendale 2004: 593597; Schrepfer-André 2005):

4.1. Cuando según encabeza un sintagma cuyo referente es una entidad de naturaleza discursiva, este aporta información acerca de las bases sobre las que se sustenta el contenido de la proposición a la que afecta. Véanse los siguientes testimonios:

(22) Según la Constitución, son las propias Cámaras las que fijan la asignación económica de diputados y senadores. (ABC, 19/09/2012)

(27) Se ve que la ley no es igual para todos. Tengo un respeto tremendo a esos afectados, pero yo tengo el mismo derecho que ellos según la Constitución. (ABC, 03/08/2010)

En ambos casos se incluye el sintagma según la Constitución, que indica en qué tipo de bases se apoya la información comunicada: tanto en (22) como en (27), el locutor se basa en un artículo que forma parte de la Constitución española. Ahora bien, en cada caso el modo de acceso a la información es diferente: en (22), el locutor se limita a hacerse eco de la información que figura en el artículo 71.4 de la Constitución española ("Los Diputados y Senadores percibirán una asignación que será fijada por las respectivas Cámaras"); en (27), en cambio, el autor del texto no extrae la información directamente de la base mencionada, sino que la construye a partir del artículo 139.1 de dicha Constitución ("Todos los españoles tienen los mismos derechos y obligaciones en cualquier parte de territorio del Estado"). Obviamente, una constitución no explicita cuáles son los derechos de un individuo concreto en unas circunstancias determinadas, sino que ofrece criterios de validez general que, posteriormente, han de aplicarse a cada situación. Esta operación exige la creación de una nueva información que, como tal, no figura en la base manejada: si la Constitución asigna los mismos derechos a todos los españoles y yo soy español, tengo los mismos derechos que otros ciudadanos espańoles afectados por un problema similar al mío cuyas solicitudes, a diferencia de las mías, sí han sido atendidas.

Así, mientras que en (22) se revela un modo de acceso reportativo a la información, en (27) el contenido de la proposición afectada por el sintagma según la Constitución es el resultado de un proceso deductivo efectuado por el propio locutor (modo de acceso inferencial; al igual, por cierto, que según el reglamento en (23) (\$2.3)). Estos testimonios ilustran cómo un mismo sintagma iniciado por según que designa un referente de carácter discursivo es compatible con diversos modos de acceso a la información, ya que únicamente introduce información acerca de las bases en que se sustenta el contenido de la proposición. 
4.2. Cuando según tiene como complemento un sintagma designador de un ser humano, este, en la mayor parte de los casos, introduce datos acerca de la identidad de la fuente de la información transmitida. Véase el caso de (21), donde el periodista se limita a recoger el contenido de un discurso del exministro de Hacienda español Cristóbal Montoro:

(21) Según Montoro, el Gobierno "ha reconocido la gravedad de la situación económica” generada por la "inactividad" del presidente del Gobierno y del vicepresidente Solbes en los últimos cuatro años. (El País, 19/04/2008)

Ahora bien, desde un punto de vista epistemológico, esta no es la única posibilidad:

(28) Si el tiempo se midiera por olimpiadas estaría a punto de vivir mi decimocuarta. Soy, según Protágoras y el poeta González Iglesias, de lo mejor que le puede pasar a unos Juegos Olímpicos; soy un contemplador. Ni negociante, ni atleta; soy el que mira. (El País, 17/07/2008)

En (28), el contenido de la proposición a la que afecta el sintagma encabezado por según no procede directamente de un discurso previo de los enunciadores citados (el filósofo Protágoras y el poeta Juan Antonio González Iglesias), sino que se trata del resultado de una inferencia efectuada por el autor del texto con base en la idiosincrasia de aquellos enunciadores: dado que es imposible que un filósofo presocrático como Protágoras haya llegado a caracterizar de una manera determinada al autor de un texto redactado en el año 2008, es obvio que, en realidad, el periodista parte de los rasgos que Protágoras y González Iglesias atribuyen a la figura del contemplador en sendas obras y, al contrastarlos con su propia personalidad, termina considerándose merecedor de tal estatus.

Algo similar ocurre en (29): al igual que en (21), en él figura el sintagma según Montoro, pero el proceso epistemológico llevado a cabo por el hablante es diferente:

(29) Montoro no les ha mandado los inspectores a los de Madrid que compraron en la reventa (tiesa) las mejores primeras filas de barrera para ver ayer a Manzanares, no. Según Montoro la crisis y el déficit se resuelven mandando inspectores de Hacienda a la Feria, pidiendo facturas en la caseta de "Amigos de las Sevillanas" o en "El Ciento y la Madre"; [...]. (ABC, 27/04/2012)

En (29), el columnista explica que los inspectores de Hacienda insistentemente han acudido a las casetas de la Feria de Abril de Sevilla en busca de irregularidades y no a otros lugares donde, en su opinión, sería esperable detectar anomalías de mayor calado. Esto le lleva a inferir, en un tono irónico, que la persona que se encuentra al mando del Ministerio de Hacienda estima que las irregularidades que pueden hallarse en esas casetas son lo suficientemente cuantiosas como para resolver todos los problemas derivados de la crisis económica en España. A juzgar por el contenido de la proposición afectada por el 
sintagma según Montoro, queda claro que el emisor no ha extraído esta información de unas declaraciones de Montoro, sino que la ha creado él mismo tras realizar una inferencia basada en las acciones efectuadas por los subordinados del ministro y, por ello, muy probablemente encomendadas por este último.

Epistemológicamente, el SN introducido por según no se corresponde ni en (28) ni en (29) con la fuente de la información. En ambos casos, el ser humano designado por ese sintagma es responsable de un discurso previo (28) o de cualquier otra acción (29) que constituye la base en que el locutor se apoya para crear una nueva información a través de una inferencia. Así pues, esa persona a la que se hace referencia no se identifica con la fuente de la información, ya que esta en ningún momento la verbaliza, y, de hecho, incluso podría oponerse a ella: por ejemplo, es verosímil pensar que Cristóbal Montoro rechazaría el punto de vista que le asigna el emisor de (29) si tuviera conocimiento de él. En todo caso, podría entenderse que la persona designada es la fuente virtual de la información, pues es el locutor y no esa otra "fuente" quien construye tal información basándose en unas palabras o acciones ajenas de las que estima que se desprende el punto de vista de esa otra persona.

Asimismo, estos testimonios muestran que ninguno de los casos de según + SN designador de un ser humano indica por sí mismo cómo ha accedido el locutor a la información, puesto que un mismo SN introducido por según puede emplearse para hacer referencia a situaciones en que la información se ha obtenido de diferentes maneras: en (21), el modo de acceso a la información es reportativo, mientras que en (29) es inferencial, pese a que en ambos casos se trate del mismo sintagma (según Montoro). Estas diferentes lecturas respecto al modo de acceso a la información son esencialmente dependientes del co(n)texto y en ningún caso vienen determinadas por defecto por el SN iniciado por según.

4.3. Con base en estas consideraciones, concluimos que no puede entenderse como evidencial ninguno de los SSNN encabezados por la preposición según que, desde una concepción más o menos aproximativa de la evidencialidad como marcación de la fuentel source o evidencialevidence, serían aparentemente evidenciales, ya que no señalan por sí mismos cuál es el modo en que el hablante ha accedido a la información, sino que aportan datos acerca de otros dos parámetros epistemológicos contiguos: la fuente y la base de la información ${ }^{24}$.

\footnotetext{
${ }^{24}$ Mención aparte merecen otros casos en que el término de según contiene ciertos verbos en forma personal, como según veo o según parece, cuyo estatus evidencial requiere una argumentación más detallada que excede los límites del presente artículo y que dejamos para futuros trabajos.
} 


\section{Conclusiones}

Muchas de las caracterizaciones de la evidencialidad más influyentes recurren a sustantivos como ingl. source, evidence o esp. fuente, evidencia sin aportar una delimitación explícita de sus referentes, pese a que, en su uso cotidiano, son capaces de designar nociones epistemológicas muy diversas. Ello ha llevado a analizar como evidenciales ciertas unidades que, en realidad, transmiten un valor muy diferente al de los morfemas evidenciales prototípicos de lenguas con evidencialidad gramaticalizada. Partiendo de estos problemas teóricos, hemos propuesto una distinción conceptual entre fuente, base y modo de acceso para discernir las nociones epistemológicas que parecen solaparse en un uso más o menos vago de los nombres sourcelfuente y evidencelevidencia, y hemos indicado que, a tenor del comportamiento de los evidenciales de lenguas con evidencialidad obligatoria, resulta más precisa una definición de la evidencialidad anclada en el concepto de modo de acceso. Esta propuesta teórica se ha aplicado al caso de los SSNN introducidos por según aparentemente evidenciales: si bien transmiten un valor próximo a la evidencialidad, que concierne o a la base de la información o a la fuente, no pueden considerarse evidenciales, ya que son compatibles con diferentes modos de acceso y, por tanto, no codifican este parámetro.

\section{Obras citadas}

Acín, Esperanza. 2014. "Prácticamente y claramente: usos estratégicos en la prensa escrita". Del discurso de los medios de comunicación a la lingüistica del discurso. Eds. Carmela Pérez-Salazar e Inés Olza. Berlín: Frank \& Timme. 175-191.

Aikhenvald, Alexandra Y. 2012. "The essence of mirativity". Linguistic Typology 16.3: 435485 .

. 2004. Evidentiality. Oxford: OUP.

2003. "Evidentiality in typological perspective". Studies in Evidentiality. Eds. Alexandra Y. Aikhenvald y R.M.W. Dixon. Ámsterdam-Filadelfia: John Benjamins. 1-31.

Anderson, Lloyd B. 1986. "Evidentials, paths of change, and mental maps: typologically regular asymmetries”. Evidentiality. The Linguistic Coding of Epistemology. Eds. Wallace Chafe y Johanna Nichols. Norwood, NJ: Ablex. 273-312.

Barrenechea, Ana Ma. 1979. "Operadores pragmáticos de actitud oracional: los adverbios en -mente y otros signos”. Estudios lingüísticos y dialectológicos. Temas hispánicos. Eds. Ma Barrenechea et al. Buenos Aires: Hachette. 39-59.

Bednarek, Monika. 2006. "Epistemological positioning and evidentiality in English news discourse: A text-driven approach". Text \& Talk 26.6: 635-660.

Bermúdez, Fernando. 2005. Evidencialidad: La codificación lingüistica del punto de vista. Tesis doctoral. Universidad de Estocolmo (Suecia).

Biber, Douglas y Edward Finegan. 1989. "Styles of stance in English: Lexical and gram- 
matical marking of evidentiality and affect", Text 9.1: 93-124.

Borillo, Andrée. 2005. "[d'après N], complément circonstanciel et marqueur énonciatif d'évidentialité". Questions de classification en linguistique: méthodes et descriptions. Mélanges offerts au Professeur Christian Molinier. Eds. Injoo Choi-Jonin et al. Berna: Peter Lang. 39-53.

Botne, Robert. 1997. "Evidentiality and epistemic modality in Lega". Studies in Language 21.3: 509-532.

Boye, Kasper y Peter Harder. 2009. "Evidentiality: Linguistic categories and grammaticalization". Functions of Language 16.1: 9-43.

Bybee, Joan L. 1985. Morphology: A Study of the Relation between Meaning and Form. Ámsterdam-Filadelfia: John Benjamins.

Chafe, Wallace. 1986. "Evidentiality in English conversation and academic writing". Evidentiality. The Linguistic Coding of Epistemology. Eds. Wallace Chafe y Johanna Nichols. Norwood, NJ: Ablex. 261-272.

Charolles, Michel. 1987. "Spécificité et portée des prises en charge en "selon A»". Revue Européenne des Sciences Sociales 25/77: 243-269.

Coltier, Danielle. 2003. "Analyse sémantique de selon. Quelques propositions (présentation de thèse)". Travaux de Linguistique 46: 117-131.

Coltier, Danielle y Patrick Dendale. 2004. "Discours rapporté et évidentialité: Comparaison du conditionnel et des constructions en selon $N$ ". Le Discours rapporté dans tous ses états. Eds. Juan Manuel López Muńoz et al. París: L'Harmattan. 587-597.

Cornillie, Bert. 2009. "Evidentiality and epistemic modality: On the close relationship between two different categories". Functions of Language 16.1: 44-62. 2007. Evidentiality and Epistemic Modality in Spanish (Semi-)Auxiliaries. A Cognitive-Functional Approach. Berlín: Walter de Gruyter.

Curnow, Timothy Jowan. 2002. "Evidentiality and me: The interaction of evidentials and first person". Proceedings of the 2001 Conference of the Australian Linguistic Society. Ed. Cynthia Allen [Recuperado de <http://www.als.asn.au/proceedings/als2001.html>].

De Bruyne, Jacques. 1999. "Las preposiciones". Gramática descriptiva de la lengua española. Coords. Ignacio Bosque y Violeta Demonte. Vol. I. Madrid: Espasa-Calpe. 657-704.

De Haan, Ferdinand. 2012. "Evidentiality and mirativity". The Oxford Handbook of Tense and Aspect. Ed. Robert I. Binnick. Oxford: OUP. 1020-1046.

. 1999. "Evidentiality and epistemic modality: Setting boundaries". Southwest Journal of Linguistics 18.1: 83-101.

DEA = Seco, Manuel, Olimpia Andrés y Gabino Ramos. 2011. Diccionario del español actual. $2^{\mathrm{a}}$ ed. Madrid: Aguilar.

DeLancey, Scott. 2001. "The mirative and evidentiality". Journal of Pragmatics 33.3: 369382.

Dendale, Patrick. 1991. Le marquage épistémique de l'énoncé: esquisse d'une théorie avec applications au français. Tesis doctoral. Universidad de Amberes.

Dendale, Patrick y Liliane Tasmowski. 2001. "Introduction: Evidentiality and related no- 
tions". Journal of Pragmatics 33.3: 339-348.

1994. "Présentation. Lévidentialité ou le marquage des sources du savoir". Langue française 102: 3-7.

DLE = Real Academia Española. 2014. Diccionario de la lengua española. $23^{\mathrm{a}}$ ed. Madrid: Espasa-Calpe.

DUE $=$ Moliner, María. 2007. Diccionario de uso del español. $3^{a}$ ed. Madrid: Gredos.

Escandell, Ma Victoria. 2010. "Futuro y evidencialidad". Anuario de Lingüistica Hispánica 26: 9-34.

Estrada, Andrea. 2008. "Ethos y discurso científico-académico. Los marcadores de evidencialidad como estrategia de cortesía”. RASAL Lingüistica 1.2: 7-24.

Faller, Martina. 2011. "A possible worlds semantics for Cuzco Quechua evidentials". Proceedings of SALT 20. Eds. Nan Li y David Lutz. Ithaca, NY: CLC. 660-683.

Féron, Corinne y Danielle Coltier. 2008. "Selon Xen français médiéval : la construction des interprétations". Congrès Mondial de Linguistique Française - CMLF'08. Eds. Jacques Durand et al. París: Institut de Linguistique Française. 173-186.

Ferrari, Laura y Susana Gallardo. 1999. "Los marcadores de evidencialidad empleados por la prensa en una controversia ambiental". Revista iberoamericana de discurso y sociedad 1.4: 69-93.

Floyd, Rick. 1997. La estructura categorial de los evidenciales en el quechua wanka. Lima: Instituto Lingüístico de Verano.

Fox, Barbara A. 2001. "Evidentiality: Authority, responsibility, and entitlement in English conversation". Journal of Linguistic Anthropology 11.2: 167-192.

Frawley, William. 1992. Linguistic Semantics. Hillsdale, NJ: L. Erlbaum.

Fuentes Rodríguez, Catalina. 2007. Sintaxis del enunciado: los complementos periféricos. Madrid: Arco/Libros.

1995. "Polifonía y argumentación: los adverbios de verdad, certeza, seguridad y evidencia en español". Lexis 19.1: 59-83.

González Vázquez, Mercedes. 2006. Las fuentes de la información: tipología, semántica y pragmática de la evidencialidad. Vigo: Universidade de Vigo.

González Vergara, Carlos. 2011. "Estrategias gramaticales de expresión de la evidencialidad en el español de Chile". Alpha: revista de artes, letras y filosofía 32: 149-165.

Guentchéva, Zlatka. 2014a. "Peut-on identifier, et comment, les marqueurs dits 'médiatifs'?". Médiativité, polyphonie et modalité en français: études synchroniques et diachroniques. Eds. Jean-Claude Anscombre, Evelyne Oppermann-Marsaux y Amalia Rodriguez Somolinos. París: PSN. 35-50.

. 2014b. "Aperçu des notions d'évidentialité et de médiativité". Cahiers de Lexicologie 105: 57-77.

1996. "Introduction". L'énonciation médiatisée. Ed. Z. Guentchéva. Lovaina-París: Peeters. 11-18.

Izquierdo Alegría, Dámaso. 2016. Alcances y límites de la evidencialidad: aspectos teóricos y propuesta de análisis aplicada a un conjunto de adverbios evidencialoides del español. 
Tesis doctoral. Universidad de Navarra (España).

Kaye, Jonathan D. 1971. "Nasal harmony in Desano". Linguistic Inquiry 2.1: 37-56.

Kim, Myong Soon. 2009. "Sur les expressions Selon + pronom personnel en français". Onŏhak, Journal of the Linguistic Society of Korea 55: 61-73.

Kronning, Hans. 2003. "Modalité et évidentialité". Aspects de la Modalité. Eds. Merete Birkelund, Gerhard Boysen y Poul Søren Kjærsgaard. Tubinga: Max Niemeyer. 131-151.

Lazard, Gilbert. 1999. "Mirativity, evidentiality, mediativity, or other?”. Linguistic Typology 3: 91-109.

Llopis Cardona, Ana. 2018. "The Spanish quotative según across written genres". Perspectives on Evidentiality in Spanish: Explorations across Genres. Eds. Carolina Figueras y Adrián Cabedo. Ámsterdam: John Benjamins. 173-204.

López Izquierdo, Marta. 2013. "Según y como: su origen y función como introductores de discurso referido". Crisol 18: 13-29.

López Martín, José Manuel. 2017. "Estudio macrosintáctico de las expresiones que introducen la fuente enunciativa: como.../ según.../ para.../ en palabras de.../ en opinión de...”. Círculo de Lingüística Aplicada a la Comunicación 71: 99-114.

Maldonado, Ricardo y Juliana De la Mora. 2015. "Según: A Space Builder into Mirativity”. eHumanista/IVITRA 8: 488-499.

Marcos Sánchez, Mercedes. 2016. "Subjetividad e intersubjetividad en los marcadores evidenciales formados por el verbo de percepción visual ver en español". La evidencialidad en español: teoría y descripción. Eds. Ramón González Ruiz, Dámaso Izquierdo Alegría y Óscar Loureda Lamas. Madrid-Fráncfort del Meno: Iberoamericana-Vervuert. 153-178.

Marque-Pucheu, Christiane. 1999. "Source, inférence et position du locuteur dans les énoncés comportant selon". Revue de Sémantique et Pragmatique 6: 103-113.

Márquez Guerrero, María. 2009. “¿Preposición imperfecta? ¿Partícula dudosa? Según: textos de los siglos XIII-XVII”. Estudios de lengua española: descripción, variación y uso: homenaje a Humberto López Morales. Eds. Ma Victoria Camacho Taboada, José Javier Rodríguez Toro y Juana de Jesús Santana Marrero. Madrid-Fráncfort del Meno: Iberoamericana-Vervuert. 439-469.

Martín Zorraquino, Ma Antonia. 1999. "Aspectos de la gramática y de la pragmática de las partículas de modalidad en español actual". Español como lengua extranjera: enfoque comunicativo y gramática. Actas del IX Congreso Internacional de ASELE. Eds. Tomás Jiménez Juliá et al. Santiago de Compostela: Universidade de Santiago de Compostela. 25-56.

Martín Zorraquino, Ma Antonia y José Portolés. 1999. "Los marcadores del discurso". Gramática descriptiva de la lengua española. Coords. Ignacio Bosque y Violeta Demonte. Vol. III. Madrid: Espasa-Calpe. 4051-4214.

Miche, Elisabeth. 2015. "An analysis of some evidential structures in essays written by students of French as a foreign language". Discourse Studies 17.2: 226-240.

Morera, Marcial. 1988. Estructura semántica del sistema preposicional del español moderno y 
sus campos de usos. Puerto del Rosario: Cabildo Insular de Fuerteventura.

NGLE = Real Academia Española y Asociación de Academias de la Lengua Espańola. 2009. Nueva gramática de la lengua española. Madrid: Espasa.

OED = Simpson, John y Edmund Weiner. 1989. Oxford English Dictionary. 2a ed. Oxford: Clarendon.

Pavón Lucero, Ma Victoria. 1999. "Clases de partículas: preposición, conjunción y adverbio". Gramática descriptiva de la lengua española. Coords. Ignacio Bosque y Violeta Demonte. Vol. I. Madrid: Espasa-Calpe. 565-656.

Pietrandrea, Paola. 2007. "The grammatical nature of some epistemic-evidential adverbs in spoken Italian”. Rivista di Linguistica 19.1: 39-63.

Plungian, Vladimir A. 2010. “Types of verbal evidentiality marking: an overview”. Linguistic Realization of Evidentiality in European Languages. Eds. Gabriele Diewald y Elena Smirnova. Berlín-Nueva York: Mouton de Gruyter. 15-58.

Rodríguez Ramalle, Teresa Ma. 2008. "El que como marca enfática discursiva en adverbios e interjecciones". 25 años de Lingüistica Aplicada en España: hitos y retos. Coords. R. Monroy Casas y A. Sánchez Pérez. Murcia: Universidad de Murcia. 811-816.

Schrepfer-André, Géraldine. 2005. La portée phrastique et textuelle des expressions introductrices de cadres énonciatifs: Les syntagmes prépositionnels en selon $X$. Tesis doctoral. Université de Paris 3 la Sorbonne-Nouvelle.

Squartini, Mario. 2004. "Disentangling evidentiality and epistemic modality in Romance". Lingua 114.7: 873-895.

. 2001. "The internal structure of evidentiality in Romance". Studies in Language 25.2: 297-334.

Thuillier, François. 2012. "Identidade e alteridade no valor da preposição selon". Revista Desenredo 8.1: 20-29.

Tournadre, Nicolas. 2004. "Typologie des aspects verbaux et intégration à une théorie du TAM”. Bulletin de la Société de Linguistique de Paris 99.1: 7-68.

Tournadre, Nicolas y Randy J. LaPolla. 2014. "Towards a new approach to evidentiality: Issues and directions for research". Linguistics of the Tibeto-Burman Area 37.2: 240-263.

Van der Auwera, Johan y Vladimir A. Plungian. 1998. "Modality's semantic map”. Linguistic Typology 2: 79-124.

Webster, Noah. 1981. Webster's Third New International Dictionary of the English Language, Unabridged. Springfield, MA: Merriam-Webster.

Whitt, Richard J. 2010. Evidentiality and Perception Verbs in English and German. Berna: Peter Lang.

Wiemer, Björn. 2010. "Hearsay in European languages: toward an integrative account of grammatical and lexical marking". Linguistic Realization of Evidentiality in European Languages. Eds. Gabriele Diewald y Elena Smirnova. Berlín-Nueva York: Mouton de Gruyter. 59-129.

Willett, Thomas. 1988. "A cross-linguistic survey of the grammaticization of evidentiality". Studies in Language 12.1: 51-97. 